\title{
Metodología de estudio de eventos como medición del impacto del dictamen del revisor fiscal en el mercado accionario colombiano en 2009-2016
}

\author{
A Methodology to Study Events as a Measuring of the Impact of the Statutory Auditor's Opinion on the \\ Colombian Stock Market during 2009-2016
}

Metodologia de estudo de eventos como aferição do impacto do parecer do revisor fiscal no mercado acionário colombiano em 2009-2016

Belky Esperanza Gutiérrez Castañeda

Universidad de Antioquia (UdeA), Colombia

belky.gutierrez@udea.edu.co

ORCID: http://orcid.org/0000-0002-9578-5968

DOI: https://doi.org/10.11144/Javeriana.cc19-47.meem Redalyc: http://www.redalyc.org/articulo.oa? id $=151556994008$

Carlos Andrés Barrera Montoya

Universidad de Antioquia (UdeA), Colombia

ORCID: http://orcid.org/0000-0002-2235-1843

Fecha de recepción: 09 Mayo 2017

Fecha de aprobación: 08 Mayo 2018

\section{Resumen:}

El fortalecimiento de un mercado accionario está relacionado directamente con la transparencia y la confiabilidad de la información disponible para los usuarios de estos mercados. Con este fin, el revisor fiscal ejerce una de sus funciones, que es el de divulgar la situación administrativa y financiera de la empresa a través del informe del revisor fiscal, convirtiéndose este informe en un garante de la realidad económica de las empresas, al verificar la ausencia de errores y fraudes en las informaciones financieras que estos usuarios reciben. A pesar de la importancia social de los revisores fiscales en el contexto colombiano, aún no se ha cuantificado el verdadero impacto de su trabajo en el mercado accionario, representado por la Bolsa de Valores de Colombia (BVC); como respuesta a la falta de esta medición, en Colombia, de la importancia e impacto que el informe del revisor fiscal pueda ejercer en el contexto empresarial y en los inversionistas, fue que se realizó esta investigación, en la cual se quiere evaluar, a través de la metodología de estudio de eventos, el efecto que el informe emitido por el revisor fiscal ejerce en relación con el dictamen de los estados financieros y su influencia en el comportamiento del precio de 127 empresas durante el periodo 2009-2016, negociadas en la BVC. Los resultados evidencian que el informe de revisoría fiscal, en conjunto con algunas variables de los estados financieros, no genera efectos significativos en los retornos esperados de las empresas que cotizan en BVC, al realizar un análisis de forma general y por sectores económicos. En conclusión, se demuestra, por medio de pruebas estadísticas, que no existe una relación significativa entre la emisión y la divulgación del informe del revisor fiscal y la volatilidad o retorno anormal que influya en la dinámica del mercado accionario.

Palabras clave: revisor fiscal, dictamen, mercado accionario, retorno de mercado, CAMP, ARCH.

\begin{abstract}
:
The strengthening of the stock market is directly related to the transparence and reliability of the information available to the users in these markets. To that end, the statutory auditor performs a particular function consisting in disclosing the administrative and financial situation of the company by means of the statutory auditor's report. It becomes a guarantee of the company financial reality as it ratifies the absence of mistakes and frauds in the financial information received by the users. Despite the social significance of the statutory auditors in the Colombian context, the true impact of their work has not been quantified so far on the stock market, which is represented by the Colombian Stock Exchange (BVC, Spanish acronym). This research was conducted in response to this measuring of the impact and significance the statutory auditor's report may have in the entrepreneurial context and the investors in Colombia. It aims to assess -through the methodology to study events- the effect of the statutory auditor's report regarding her/ his professional opinion about the financial statements and the influence thereof on the price behavior of 127 companies during 2009-2016, which were traded in the BVC. The results indicate that the statutory auditor's report together with some variables in the financial statements do not bring about any significant effects on the return expected by the companies trading in the BVC, as found in the analyses both general and per economic sector. In conclusion, the statistical evidence demonstrates that there is not any significant relationship between the issuing and disclosing the statutory auditor's report and the abnormal return or volatility that would impact the stock market dynamics.
\end{abstract}

Keywords: statutory auditor, professional opinion, stock market, market return, CAMP, ARCH. 


\section{Resumo:}

O fortalecimento de um mercado acionário está relacionado diretamente à transparência e a confiabilidade das informações disponíveis para os usuários nesses mercados. Com esse fim, o auditor exerce uma de suas funções, que é a de divulgar a situação administrativa e financeira da empresa através do relatório do auditor, virando este relatório um fiador da realidade econômica das empresas ao verificar a ausência de erros e fraudes nas informações financeiras que esses usuários recebem. Embora a importância social dos auditores no contexto colombiano, ainda não tem-se quantificado o verdadeiro impacto do seu trabalho no mercado acionário, representado pela Bolsa de Valores da Colômbia (BVC); como resposta à falta dessa medição na Colômbia, da importância e impacto que o relatório do auditor pode exercer no contexto empresarial e nos investidores, foi que esta pesquisa foi feita, na qual quer-se avaliar, através da metodologia de estudo de eventos, o efeito que o relatório emitido pelo auditor exerce em relação com o parecer das demonstrações financeiras e sua influência no comportamento do preço de 127 empresas durante o período 2009-2016, negociadas na BVC. Os resultados mostram que o relatório de auditoria, juntamente com algumas variáveis das demonstrações financeiras, não gera efeitos significativos nos retornos esperados das empresas listadas na BVC, ao realizar análise de forma geral e por setores econômicos. Em conclusão, demonstra-se, por meio de dados estatísticos, que não existe relação significativa entre emissão e divulgação do relatório do auditor e a volatilidade ou retorno anormal que influencia a dinâmica do mercado acionário.

Palavras-chave: auditor, parecer, mercado acionário, retorno de mercado, CAMP, ARCH.

\section{Introducción}

La existencia de diversos agentes económicos dentro del funcionamiento de las organizaciones genera una serie de conflictos enmarcados en los diferentes intereses, dando lugar a problemas que recurrentemente afectan el desarrollo de las actuales organizaciones, como el desvío de recursos o fraudes, la ineficiencia de los procesos organizacionales y el incumplimiento de las normas vigentes en las empresas y su medio externo. Por tanto, ante estos conflictos de intereses entre los participantes del mercado y de asimetrías de información, la auditoría emerge ante la necesidad de evaluar la calidad y la veracidad de la información económica financiera de un negocio que se debe ejercer de forma independiente, para que así los agentes del mercado tomen decisiones acertadas. De allí su importancia y contribución, para que los usuarios de la información desarrollen de manera eficiente y eficaz sus negociaciones y reduzcan el riesgo de operar con información errada que no refleja la realidad del entorno económico.

En el caso colombiano, ante esta necesidad de divulgar una información confiable surge la revisoría fiscal como una figura que representa un instrumento de fiscalización, inspección y vigilancia de las sociedades mercantiles, por medio de la cual el Estado controla, de manera política y social, a las organizaciones, interviene en los sectores de la economía a partir del informe que emite el revisor fiscal que, en representación del Estado, será el garante de dar fe pública de los hallazgos identificados como resultado del proceso de fiscalización ${ }^{[1]}$ realizado a las empresas del sector privado (Mantilla, 2003).

En el contexto internacional, diversos investigadores han evaluado la relación entre el dictamen del auditor y su impacto en el mercado accionario, como los casos de los estudios realizados en los mercados accionarios de Estados Unidos, Europa y Asia, en los cuales se han encontrado conclusiones contradictorias en contextos económicos similares. En el caso europeo, Soltani (2000) evaluó al mercado francés utilizando 543 dictámenes de auditoría en el periodo comprendido entre 1986 y 1995, en tres diferentes fechas de eventos y con cinco modelos de mercado, e identificó que este mercado presentó una volatilidad accionaria al conocer o salir a la luz pública el informe del auditor, provocando un retorno anormal positivo o negativo accionario; caso contrario, y en el mismo contexto económico europeo, es el estudio realizado por Martínez et al. (2004) al mercado español, con el que identificó que en este mercado no se presentó un retorno anormal o una volatilidad en el precio de las acciones, ante la divulgación del informe de auditoría de las empresas auditadas.

Por otro lado, otras investigaciones se han enfocado en el mercado chino, como las de Defond y Wong (2000); Chen, Su y Zhao (2000); Haw, Qi y Wu (2003); Lin, Tang y Xiao (2003, p. 3) y Pei, Opong y Hamil (2004); igual en los mercados europeos y de Estados Unidos, con los estudios de Choi y Jeter (1992); Chen et al. (2000) y Ameen et al. (1994). Debemos aclarar que en el caso colombiano no existen, en la literatura, 
estudios que relacionen el dictamen del auditor con el mercado accionario colombiano representado por la BVC, específicamente, un estudio que analice el impacto que pueda generar el informe del revisor fiscal en estos mercados, considerando que él es innato al contexto colombiano, y como se ha expresado anteriormente, se trata de un organismo de control que permite inspeccionar los estados financieros y las acciones de los administradores de las empresas, dando fe pública de sus actuaciones y registros.

A raíz de este contexto, podemos extrapolar en Colombia los resultados obtenidos en otros mercados; considerando que en el país existen las figuras del revisor fiscal y del auditor, ¿cuál de ellos, al emitir su dictamen e informe, impacta la volatilidad accionaria, provocando un retorno anormal? Buscando evaluar este interrogante y cuantificar la relación del informe del revisor fiscal y el precio de las acciones, surgen las siguientes preguntas:

1. Si la revisoría es un factor importante para mantener la credibilidad en las informaciones contables y en la administración, ¿los usuarios del mercado accionario colombiano consideran el trabajo del revisor fiscal igualmente importante?

2. Si diferentes estudios realizados en Europa, Asia y Norteamérica muestran resultados que permitan su generalización o extrapolación a otros mercados, ¿entonces los usuarios del mercado accionario colombiano deben continuar usando esas conclusiones ajenas al contexto de nuestro país?

Las respuestas a estas preguntas no solo justifican la relevancia del tema investigado (cuantificar la relación revisoría fiscal-mercado accionario o informe del revisor fiscal-precio de las acciones), sino que también dimensionan y resaltan la importancia de la información contable y su complejidad en el proceso de decisión de los inversionistas en los mercados accionarios. El objetivo general en esta investigación es evaluar la relación existente entre la divulgación de los informes de revisoría fiscal y el comportamiento de los precios de las acciones de las empresas que cotizan en el mercado accionario colombiano, específicamente en la BVC. Para esto se utiliza la metodología de estudios de eventos Fama (1991), en la cual se busca determinar el impacto de determinado acontecimiento; en este caso, el evento estudiado se representa por la fecha de la divulgación de los informes del revisor fiscal y de los estados financieros de las empresas que participan o participaron en la BVC.

Estos efectos se evalúan por medio de la variación o volatilidad de los precios de las empresas que cotizan o cotizaron en la bolsa de valores entre los años 2009 y 2016. En consecuencia, esta metodología pretende encontrar retornos anormales en los precios asociados al evento; en este sentido, se desea verificar si existe una variación anormal en el precio de una acción, luego de conocerse una información positiva o negativa de los informes del revisor fiscal.

A partir de los retornos normales y de los retornos estimados se calculan los retornos anormales acumulados (CAR, por sus siglas en inglés), realizando un análisis con una ventana de evento de CAR (-2,2), CAR $(-3,3)$ y CAR $(-5,5)$, y siguiendo esta metodología utilizada en otros estudios, los cuales tenían el mismo objetivo (Bollerslev, 1986; Chiang y Doong, 2001). Igualmente, al utilizar el modelo de mercado por medio de regresiones lineales, que no presenta un comportamiento lineal sino cuadrático, en este caso se utiliza el modelo Autoregressive Conditional Heteroskedasticity (ARCH) para estimar los retornos esperados de las empresas, ya que se identificó que no todas, en su valor accionario, muestran un comportamiento lineal. En consecuencia, se aplica la prueba, según Engle (1982), en las regresiones de cada uno de los CAR. Así se realiza entonces un análisis univariado entre la variable CAR, para cada una de las ventanas de estimación, donde se exploró la correlación entre el informe del revisor fiscal y las variables financieras, como las siguientes: 1) la ganancia o pérdida; 2) la rentabilidad de las acciones; 3) la utilidad por acción; 4) los dividendos por acción; 5) el total de activos; 6) la valorización de los activos; 7) el índice Book to Market, entre otras. Este modelo se analiza de forma general entre los años 2009 y 2016 y por sectores económicos y se encuentra principalmente que la divulgación y la información contenida en el informe de revisoría fiscal divulgado por las empresas que cotizan en la BVC, no interfiere en el precio accionario y, por consiguiente, en este trabajo podemos afirmar 
que, por pruebas estadísticas, el trabajo del revisor fiscal en Colombia, concretizado en el informe, no incide en la dinámica de este mercado.

\section{Revisión de la literatura}

Escándalos financieros de carácter nacional e internacional caracterizados por la corrupción, desfalcos y delitos cometidos por funcionarios de alto nivel dentro de las organizaciones, justifican la existencia de una práctica sistemática que al amparo del carácter de independencia mental y profesional haga seguimiento y evalúe los procesos que llevan las empresas, para garantizar la confiabilidad y razonabilidad de la información y, por ende, una toma de decisiones más acertada de los diferentes usuarios de la información financiera.

\section{La revisoría fiscal en Colombia}

Teniendo en cuenta lo anterior, cabe complementar que en Colombia, la revisoría fiscal y la auditoría son un instrumento de control que se encuentra reglamentado en la normatividad colombiana. En este sentido, los siguientes son algunos de los acontecimientos que estimularon la preponderancia de la auditoría en Colombia (Tabla 1): 
TABLA 1

Acontecimientos que estimularon la preponderancia de la auditoría en Colombia, 1983-1987

E1 Instituto de Seguros Sociales empezó a realizar marcos teóricos sobre

1983-1987

la calidad en los servicios de salud, según el documento Garantía de los

servicios de salud.

\begin{tabular}{ll}
\hline & La reforma constitucional de 1991, en sus artículos 209 y 269 estableció \\
& el deber de la administración pública de diseñar y aplicar métodos y \\
& procedimientos de control cuya calidad y eficiencia será evaluada por la \\
& Contraloría General de la Nación. \\
\hline Ley 87 de 1993 & Implementa, desarrolla y consolida las auditorías internas en el sector \\
& estatal; su filosofia y enfoque es retomar del sector privado la práctica de \\
& la auditoría, el control interno y otras herramientas de tipo gerencial. \\
\hline Ley 100 de 1993 & Inicia en Colombia un proceso de mejoramiento continuo de la calidad en \\
la prestación de los servicios de salud, donde la auditoría cumple un papel & protagónico en la verificación permanente del cumplimiento de los \\
& estándares de calidad establecidos por el gobierno nacional. \\
\hline Se establece la calidad como fundamento del servicio público y se \\
determina que el sistema establecerá mecanismos de control a los servicios \\
para garantizar a los usuarios calidad en la atención oportuna, \\
personalizada, humana, integral, continua y de acuerdo con los estándares \\
aceptados en procedimientos y prácticas profesionales. De acuerdo con la \\
reglamentación que expida el gobierno, instituciones prestadoras deberán \\
estar acreditadas ante las entidades de vigilancia \\
\hline 153 de la Ley 100 de
\end{tabular}

Fuente: elaboración propia.

Igualmente, la Ley Sarbanes Oxley (2002) define la auditoría como un "examen de los estados financieros de cualquier emisor realizado por una firma de auditoría independiente, de acuerdo con las reglas de la Junta o de la Comisión, con el propósito de expresar una opinión sobre tales estados".

Por lo anterior, la auditoría en Colombia y en el resto del mundo pasa a ser hoy una herramienta que permite no solo la detección de fraudes en una entidad (privada, pública o mixta), sino que mejora la credibilidad de los procesos contables y administrativos, además de verificar si estos últimos se están ejecutando de acuerdo con la normatividad vigente. Sumado a lo anterior la existencia de un escenario económico en el que se genera gran cantidad de información, se requiere de mecanismos que fomenten la construcción de un ambiente 
de confianza que permita el adecuado funcionamiento de las organizaciones. Por ello, la revisoría fiscal en Colombia es un elemento determinante que permite dar credibilidad a la información generada por las empresas y logra el desarrollo de la economía de mercado, ya que disminuye el riesgo de que exista información errónea o ficticia que lleve a tomar decisiones equivocadas en desarrollo de los negocios.

Dentro de la complejidad del contexto local e idiosincrático colombiano existe la figura de la revisoría fiscal, que representa una institución de control que tiene su origen en la corriente de control latina, mediante la cual el Estado controla de manera política y social a los ciudadanos y a las organizaciones, es decir, interviene las operaciones del sector privado por medio de un contador público (revisor fiscal), quien en representación del Estado tiene la facultad de brindar fe pública de los hallazgos que aparezcan como resultado del proceso de fiscalización a las entidades del sector privado. Este modelo de control traza una dicotomía significativa con el modelo de control anglosajón, ya que en este último prima la libertad de empresa con el mínimo de intervención estatal. La revisoría fiscal ha sido una de las armas para combatir el delito y la corrupción empresarial, preocupantes en el contexto colombiano, no solo en el sector privado, sino en el público. Igual que la auditoría, la revisoría fiscal debe actuar con independencia mental, política y económica y hacer un examen integral a las organizaciones, para crear una comunicación objetiva con el Estado y mejorar la relación empresa-Estado.

Es importante reiterar que cuando se habla de dictamen de auditoría o del informe del revisor fiscal se hace referencia a la auditoría externa y a la revisoría fiscal, que son las únicas facultadas en Colombia para dictaminar y evaluar los estados financieros de las empresas y los procesos de la administración. Así mismo, el informe del revisor fiscal es un documento con ciertos parámetros y especificaciones en el que este emite su opinión, de manera técnica y experta, a partir de los resultados de su revisoría, que en el caso de la auditoría financiera dictaminará respecto a la razonabilidad de los estados financieros. Tal opinión se emite de acuerdo con la normatividad vigente para la preparación de estados financieros. De igual forma, la opinión del revisor fiscal como resultado de su trabajo y expresado en el informe requiere de los garantes de la credibilidad de la información emitida por la empresa un plan de trabajo bastante juicioso y responsable. Tal como lo señala el estándar internacional de auditoría 330 (planificación de la auditoría de estados financieros), el auditor debe obtener una evidencia de auditoría para expresar conclusiones razonables en las cuales fundamentar la opinión de su auditoría (Blanco, 2004).

\section{La auditoría y el mercado accionario}

Estudios empíricos realizados en diferentes mercados accionarios han identificado que en algunos casos existe una relación directa entre la divulgación del dictamen de auditoría y la volatilidad accionaria [Defond y Wong (2000); Chen et al. (2000); Haw et al. (2003); Lin et al. (2003, p. 6) y Pei et al. (2004)]; igual que en los mercados europeos y de Estados Unidos [Choi y Jeter (1992); Chen et al. (2000) y Ameen et al. (1994)]. En algunos casos, utilizan información que contiene los estados financieros para comprobar por medio de modelos matemáticos la relación directa de tales ítems y darle más fuerza o caracterización al análisis de la volatilidad accionaria.

Ante la dinámica del actual entorno económico, el mercado accionario se ha convertido en un importante canalizador de recursos para el desarrollo del actual sistema económico. Es el medio por el cual circulan los flujos de fondos que los entes económicos requieren para su sostenibilidad y para ejecutar nuevos proyectos de inversión en aras de alcanzar un mayor crecimiento económico. Estos flujos de fondos pueden ser adquiridos, si bien de manera interna mediante los recursos disponibles por la generación de flujos de caja, como resultado de las operaciones de la empresa o generalmente se requiere de fuentes externas ligadas al mercado accionario con la emisión de títulos; usualmente por medio de acciones. Sin embargo, el comportamiento de esas acciones no solo depende de los informes contables, sino que también puede estar afectado por diversos factores económicos que cambian sus precios y su aceptación del público en un mercado accionario (Teweles 
et al., 1992). En este sentido, según Bromwich (1992), los estados financieros y el dictamen de auditoría de las empresas de sociedad anónima contienen información relevante para la toma de decisiones de los usuarios de cualquier mercado accionario.

Autores clásicos como Ball y Brown (1968), Bernard y Thomas (1990) y Jegadeesh y Livnat (2006) afirman que existe considerable evidencia que apoya la correlación existente entre la información de la utilidad de las empresas y la variación de los precios de acciones (Lev, 1989). Igualmente, según Choi y Jeter (1992), el informe de auditoría es la fuente de información más importante, dado que este tiene el poder de cambiar la respuesta del mercado a las ganancias, es decir, ella puede afectar significativamente el precio de las acciones. Esto último se puede ver reflejado en los flujos de efectivo; el informe de auditoría puede tener información vital sobre la viabilidad y la continuidad de la empresa (información privilegiada y acceso a información privada).

Otros autores han intentado responder a si los informes de auditoría afectan el mercado accionario, provocando la volatilidad de los precios de las acciones, por lo que este concepto entra en controversia. Estudios clásicos como los de Loudder et al. (1992) y Jones (1996) consideran directamente su impacto sobre la rentabilidad de los títulos, y otros solo analizan algún tipo concreto de calificación o informe (Dodd $e t$ al., 1984); igualmente, sobre opiniones de auditoría negativas y con salvedades, García (2000) muestra que estos informes producen muy poco efecto sobre el mercado; Chow y Rice, (1982a) revelan que los informes de auditoría con salvedades transmiten información adversa, que se asocia con la disminución del precio de las acciones. Ameen et al. (1994) defienden la idea de que el mercado sí responde a los sucesos de opiniones de auditoría distintas de las favorables. Así mismo, Melumad y Ziv (1997) proponen un modelo que mide las reacciones del mercado ante dos tipos de dictámenes y muestra que este reacciona de manera diferente ante los informes de auditoría con salvedad. Por último, algunos de los estudios anteriormente mencionados, realizados para el mercado accionario americano y otros, utilizan la metodología de eventos, como el del mercado de Londres (Firth, 1978); sobre el mercado español están los estudios de Martínez et al. (2004); sobre el mercado asiático, Chen et al. (2000), y Knechel y Sharma, (2012) sobre el mercado hindú. Todos ellos presentan diferentes tipos de dictámenes de auditoría.

\section{Metodología de la investigación}

La metodología de estudio de evento se ha utilizado generalmente para observar la reacción del mercado ante un evento específico o para cuantificar el impacto de un evento en la riqueza de los accionistas (Berggrun, 2006), por lo cual, su evolución ha estado ligada al desarrollo organizacional y del mercado de capitales del último siglo, que se inicia con los primeros estudios publicados a principios del siglo XX. El trabajo de Dolley (1933) se considera el primer ejemplo de estudio publicado sobre esta metodología.

Posteriormente, durante los siguientes treinta años se observa un importante avance en la aplicación de la metodología en trabajos como los de Myers et al. (1948), Barker (1956, 1957, 1958) y Ashley (1962), que dan tratamiento al movimiento de los precios de las acciones y a los efectos perturbadores. Finalizando la década de los años sesenta, Ball y Brown (1968) y Fama (1969) desarrollan esta metodología, que se asemeja a la actualmente utilizada. Para inicios de los años noventa, con el trabajo de Eugene Fama (1991) esta metodología se empieza a utilizar en el análisis de mercados eficientes y en el impacto de los eventos en el precio de las acciones. Dentro de este desarrollo histórico, a partir de la década de los años setenta, se da inicio a una serie de trabajos que busca dar solución a problemas evidenciados en los primeros estudios.

En el caso colombiano ha existido poca aplicabilidad de esta metodología, dado que recién inicia su difusión. Algunos estudios de los últimos años son los de Berggrun (2006), Velásquez (2009), Gómez (2009) y Gutiérrez (2011), que han realizado investigaciones referentes al mercado accionario colombiano, evaluando diferentes impactos y utilizando diversas variables, por medio del estudio de eventos. 
El avance de la literatura empírica ha permitido comprobar que los estudios orientados a determinar la importancia de la opinión del auditor y del revisor fiscal para los inversionistas, depende de los informes de auditoría comprobados con la metodología de eventos. Así, esta investigación se desarrolló con un enfoque cuantitativo y con un alcance descriptivo y correlacional, en el que se utilizaron herramientas contabilométricas, modelos matemáticos y estadísticos, desarrollando cálculos a través de regresiones lineales simples, para obtener resultados que nos llevaran a determinar si los retornos eran normales o anormales; por otro lado, con el fin de ampliar el tamaño de las observaciones o muestras se utiliza el método de datos de panel con corte transversal, que amplía nuestras observaciones en el periodo de tiempo analizado de 2009 a 2016.

Para definir la base de datos que se utilizó se consideraron los siguientes aspectos fundamentales: 1) el número de empresas y 2) el periodo que se tratará en el trabajo. En cuanto al número de empresas, el objetivo fue analizar las que cotizan o cotizaron en la BVC en el periodo de estudio; así, por medio de datos de panel y considerando los criterios poblacionales, se comenzó con una población de 63 empresas, obteniendo un total de 504 observaciones (población). Los criterios poblacionales establecidos con el fin de cumplir con el objetivo de investigación fueron los siguientes: 1) que el precio accionario de las empresas (observaciones) presente su valor continuo durante la ventana de evento y estimación; para esto se utilizan datos obtenidos del sistema Economática; 2) se utilizará solo el precio de las acciones de valor ordinario; aplicando los criterios anteriormente mencionados se obtuvo un total de 320 observaciones; los otros criterios considerados principalmente de información financiera son: 3) total de activos; 4) capitalización bursátil; 5) apalancamiento financiero; 6) dividendos por acción; 7) utilidad por acción, y 8) ganancias o pérdidas totales. Estas últimas variables se obtuvieron también de Economática, además de la página web de la Superintendencia Financiera de Colombia y el enlace de las empresas evaluadas. Se obtuvo un total de 156 observaciones que cumplen con los criterios establecidos para la muestra poblacional.

El último cálculo realizado con el fin de determinar el total de la muestra fue el cálculo de los retornos anormales (CAR), de una nuestra variable dependiente e identificando como día de evento la fecha de divulgación del informe del revisor fiscal; según MacKinlay (1997), para determinar el día del evento se deben considerar las siguientes características:

1. El día del evento: se consideró el día de divulgación de los informes de gestión de las empresas que cotizan en la Bolsa de Valores de Colombia en el periodo de estudio y se analizó principalmente la fecha de la firma del informe del revisor fiscal. Esta información sale a la luz pública entre los meses de abril, mayo y junio del año siguiente de los años de interés, es decir, para una empresa perteneciente a la BVC, la información financiera y el informe de revisoría fiscal del año $t$ está disponible en alguno de los meses anteriormente descritos del año $t+1$.

2. Ventana de evento: esta es de una longitud de 5,7 y 11 días, en las cuales se incluye el día del evento. Es decir, consideraremos 2, 3 y 5 días antes del día del evento y 2, 3 y 5 días después del CAR $(-2,2)$, CAR $(-3,3)$ y CAR $(-5,5)$, para formar así tres retornos anormales acumulados y determinar con mayor exactitud la variación o retorno anormal que el informe del revisor fiscal puede ocasionar a las empresas analizadas, considerando diferentes ventanas de eventos.

3. Ventana de estimación: se toma un lapso de 60 días para cada una de las ventanas de eventos descritas en el apartado anterior. Aquí se eliminan aquellas empresas que tengan menos de 30 días de cotización en la ventana estimación, durante el periodo de la muestra, con el fin de crear sesgo en la estimación de los parámetros. En total, la muestra final está conformada por 127 empresas, durante el periodo de 2009 a 2016, divididas en seis sectores económicos, según clasificación de la BVC (comercial, financiero, industrial, inversiones, público y servicios). 


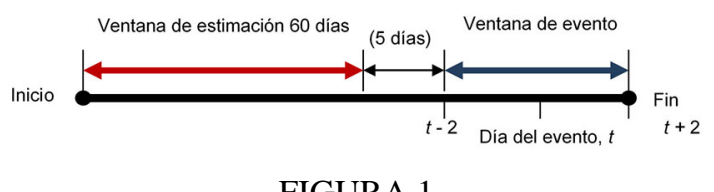

FIGURA 1

Diagrama de estudio de evento.

\section{Modelo de mercado}

Para calcular la variable dependiente CAR de nuestro modelo y encontrar el alfa y el beta, se seleccionó el modelo económico tradicional, realizando una regresión para los días comprendidos en el periodo ajeno al evento o periodo limpio:

$$
\begin{aligned}
& R_{i t}=\alpha_{i}+\beta_{i} R_{\mathrm{mt}}+e_{i t}(1) \\
& E\left(\varepsilon_{i}\right)=0 \text { y } \operatorname{Var}\left(\varepsilon_{i t}\right)=\sigma_{i t}^{2}
\end{aligned}
$$

Donde:

$R_{i t}$ INSERTAR EC 5: es el retorno de la acción i

$R_{\mathrm{m}}$ : es el retorno del índice de mercado para el día t.

$\beta_{i}$ : es la sensibilidad de los retornos de la empresa ante los retornos del mercado (pendiente)

$\alpha_{i}$ : representa los retornos que no logra explicar el modelo (intercepto)

$e_{i t}$ : error estadístico con valor esperado de cero

De esta regresión se obtienen los estimadores de cada uno de los parámetros, que son la base fundamental para el retorno esperado en la ventana del evento:

$$
\widehat{R_{i t}}=\widehat{\alpha}_{i}+\widehat{\beta}_{i} R_{\mathrm{mt}}
$$

Donde $R_{\mathrm{mt}}$ ahora es el retorno (diario) del índice de mercado en el periodo del evento. Berggrun (2006) define un test que se aplica cuando el modelo de regresión utiliza valores estimados por mínimos cuadrados ordinarios, como el dado por la ecuación 1 (modelo de mercado); se transgrede el hecho de que los residuales de la regresión se distribuyen en i.i.d N $\left(0, \sigma^{2}\right)$ Ahora, si los residuales del modelo se distribuyen de forma normal, pero con varianza no constante, el modelo de regresión se debe estimar nuevamente, según el modelo de mercado de Bollerslev (1986) y Chiang y Doong (2001), pero ya utilizando los modelos ARCH o Generalized Autoregresive Heteroscedastic (Garch), dados por la siguiente ecuación:

$$
\begin{gathered}
R_{i t}=\alpha_{i}+\beta_{i} R_{\mathrm{mt}}+e_{i t}(2) \\
e_{i t} \text { i.i. } d \sim N\left(0, \sigma^{2}\right)
\end{gathered}
$$

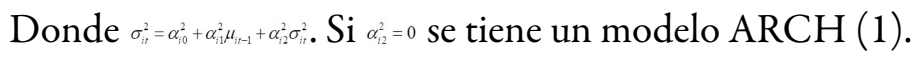

Así, el retorno anormal es una variable que se define en términos generales como la diferencia entre el retorno observado del activo en la ventana del evento y el retorno esperado de la acción específica si el evento no tuviera lugar; entonces, este retorno es el que calcula cuándo el evento tiene lugar. Podemos definir entonces una ecuación que nos represente el retorno anormal, pues ya se tiene calculado el retorno esperado por cada uno de los métodos anteriormente explicados, si llamamos a $R_{t}$ como el resultado o el residuo de esta diferencia para cada día de la ventana. Según MacKinlay (1997), este será igual al retorno observado $R_{t}$ , menos el retorno esperado $\overline{R_{t}}$, 


$$
r_{t}=R_{t}-\widehat{R_{t}}(3)
$$

El residuo mostrado en la ecuación tres representa el retorno anormal; en otras palabras, el retorno que no se espera y que representa un estimado del cambio en el valor de la firma, debido al evento. A este retorno anormal también se le conoce comúnmente como Anormal Return (AR, por sus siglas en inglés) (Berggrun, 2006). Ahora, el CAR es la suma de todos los retornos anormales durante la ventana de evento; un CAR positivo indicará que el suceso estudiado tuvo un impacto positivo sobre el precio de las acciones, y un CAR negativo indicará que el suceso tuvo impacto y provocó una volatilidad en el precio de las acciones. Así, esta investigación establece como variable dependiente al CAR. En forma general, el CAR es expresado por MacKinlay (1997) de la siguiente manera (ecuación 4), para $\tau_{1}$ a $\tau_{2}$ donde $\mathrm{T}_{1}<\tau_{1} \leq \tau_{2} \leq \mathrm{T}_{2}$.

$$
\operatorname{CAR}\left(\tau_{i}, \tau_{2}\right)=\sum_{r=r_{1}}^{\tau_{2}} r_{t i}
$$

\section{Donde:}

- $\quad r_{i t}$ son los retornos anormales de la empresa i.

- $\quad \tau_{1}$ y INSERTAR $\tau_{2}$ son los límites inferior y superior de la ventana de evento.

- Asintóticamente, a medida que aumenta (ventana de estimación), la varianza del $C A R_{i}\left(\tau_{1}, \tau_{2}\right.$ está dada:

$$
\sigma_{i}^{2}\left(\tau_{1}, \tau_{2}\right)=\left(\tau_{1}-\tau_{2}+1\right) \sigma_{\varepsilon i}^{2}
$$

Cada uno de estos retornos anormales se obtiene como la diferencia entre el valor del retorno normal y el retorno esperado en el día $t$ (donde t es un día perteneciente a la ventana de evento). Ahora, para el cálculo de los retornos esperados en el día usamos el modelo de mercado descrito por la ecuación tres, donde la variable dependiente de este modelo son los retornos de mercado de las empresas $\left(R_{p, t}\right)$ y la variable independiente es el retorno de los retornos de mercado $\left(R_{m, t}\right)$; cada uno de estos retornos se calcula utilizando la siguiente fórmula: $\left(p_{p m s}=\frac{p_{p+1}-P_{i}}{p_{i}}\right)$, donde $P_{t+1}$ e $P_{t}$ representan los precios de las acciones o del índice de mercado en los días $t+1$ e $t$ de cotización durante los días de la ventana de estimación.

La metodología de esta investigación se desarrolló centralmente en la de estudio de eventos, que ha sido muy utilizada en estudios que proponen el análisis del impacto en el comportamiento de los precios de las acciones ante la ocurrencia de un evento dado. Este método es desarrollado por Ball y Brown (1968), y Fama (1969), y su propósito principal es cuantificar la reacción de los mercados financieros -en general, en corto plazo - ante anuncios/eventos relacionados con alguna empresa; el evento para analizar es la publicación del informe del revisor fiscal de las empresas que cotizan o cotizaron en la BVC, para lo cual se requiere definir el método investigativo, las herramientas utilizadas para seleccionar la muestra y las fuentes de información, que en este caso fueron los siguientes:

1. La investigación es deductiva, dado que se quiere evaluar el comportamiento del precio de las acciones durante un periodo específico, y se quiere establecer si la volatilidad de las acciones se afecta con la emisión del informe del revisor fiscal en Colombia.

2. La investigación es cuantitativa, aunque se utilizarán variables de corte cualitativo que serán evaluadas por medio del tratamiento de variables Dummy.

Para verificar el impacto de los informes de revisoría fiscal en el mercado accionario colombiano nos planteamos las siguientes hipótesis, motivadas por la evidencia empírica y los hallazgos de las decisiones de inversión de ciertas carteras:

$\mathrm{H}_{\mathrm{i}}$ : el informe del revisor fiscal impacta en la volatilidad del precio accionario, provocando retornos anormales acumulados en las acciones de las empresas de la BVC. 
De lo anterior, podemos concluir que la hipótesis nula corresponde a la siguiente:

$\mathrm{H}_{0}$ : El informe del revisor fiscal no impacta en la volatilidad del precio accionario, es decir, no produce retornos anormales acumulados en las acciones de las empresas de la BVC.

\section{Análisis de los resultados}

Utilizando el modelo de Regresión lineal se quiso sustentar la hipótesis de investigación, considerando a este modelo como una técnica utilizada por distintas disciplinas de la ciencia para investigar y modelar relaciones entre variables; para este estudio se establecerán las siguientes variables:

$\mathrm{CAR}_{\mathrm{ij}}$ : definida anteriormente, representa la variable dependiente en un modelo de regresión lineal, es la rentabilidad anormal acumulada de las ventanas de estimación de longitudes de 5,7 y 11 días para que la empresa $i=1,2, \ldots 127$ en la fecha de presentación de las cuentas anuales, informe de auditoría para el año fiscal $j=2009, \ldots, 2016,\left[\operatorname{CAR}_{i, j}(-2,2)\right],\left[\operatorname{CAR}_{i, j}(-3,3)\right],\left[\operatorname{CAR}_{i, j}(-5,5)\right]$, con una ventana de estimación de 60 días.

D_IRF: es la variable Dummy que toma el valor de 0 (cero) en los informes de revisoría fiscal modificados con salvedad, y 1 (uno) para los informes de auditoría sin modificar o limpios.

Igualmente, se utilizan variables de control que son el grupo para el cual no hay intervención; en este grupo se compara al grupo que experimenta la intervención y a la diferencia de los resultados del grupo atribuidos al efecto de la intervención. Entonces, debemos incluir variables que permitan controlar la expectativa previa del mercado en relación con el informe del revisor fiscal. Como en Krishnamurthy et al. (2006), se incluyen variables como la tasa book to market, el apalancamiento financiero, el tamaño de la empresa y las ganancias y pérdidas, entre otras:

1. Apal_f: variable de control de tipo cuantitativa que representa la ratio de apalancamiento de las empresas.

2. G_P: variable de control que se define en este estudio como variable Dummy, que toma el valor de uno (1) para las empresas que presentan lucro negativo en el año en curso y de cero (0) en caso contrario. Algunos estudios han mostrado que las divulgaciones de las ganancias contables tienen efecto sobre el precio de las acciones (Ball y Brown, 1968; Elliot, 1982; Haw et al., 2000, y Gutiérrez, 2011).

3. $\quad \ln (\mathrm{TA})$ : tamaño de las empresas; su medición puede adoptar diferentes técnicas, entre el principal (1), el número de empleados, y 2, el valor y/o el número de activos de la compañía al final de año (Dhawan, 1999; Gutiérrez, 2011). Para el mercado accionario colombiano es más fácil hallar la información del total de activos de las empresas. Por tanto, para este modelo se utiliza la variable normalizada mediante el logaritmo natural de los activos.

4. VA: variable que se utiliza para observar el comportamiento o las fluctuaciones del precio de las acciones, refleja la tasa de crecimiento, la cual parte de dividir la diferencia entre el precio $(t)$ y $(t-1)$ con respecto al precio del día $(\mathrm{t}-1)$.

5. Div: variable que mide el cambio porcentual o la tasa de crecimientos en los pagos de dividendos entre los periodos $(t)$ y $(t-1)$. Según la teoría, iniciada por Black y Scholes (1974), atribuye las fluctuaciones en los precios de las acciones durante los periodos de pago de dividendos.

6. Upa: utilidad por acción definida por Brown et al. (1987) como la diferencia entre las utilidades reales y las esperadas. Se construye como su tasa de crecimiento y parte de dividir la diferencia entre la utilidad por acción esperada en el tiempo $(\mathrm{t})$ y $(\mathrm{t}-1)$, de acuerdo con la utilidad por acción del día $(\mathrm{t}-1)$.

7. Ren_A: es la relación entre el ingreso anual sobre los activos totales del año anterior. 
8. DeltaVol: es el cambio en el volumen de operaciones promedio de la empresa después de dividir por su activo total, como se muestra en la siguiente igualdad:

$$
\text { DeltaVol }=\frac{\text { volumen }_{t}}{\text { total activo }_{t}}-\frac{\text { volumen }_{t-1}}{\text { total activo }_{t-1}}
$$

\section{Análisis descriptivo del modelo general}

Cada CAR calculado para este estudio se estima por mínimos cuadrados ordinarios (MCO), por medio del modelo de mercado dado por la ecuación 1, utilizando los datos de los 60 días de la ventana de estimación; en este caso se tomó el retorno de mercado (variable independiente), los retornos en tiempo continuo del índice de mercado accionario Colcap y, como variable dependiente, los retornos en tiempo continuo de cada una de las 127 empresas de la muestra. También en este caso, teniendo como base los estudios realizados por Bollerslev (1986) y Chiang y Doong (2001), se consideró la posibilidad de la existencia de efectos ARCH en los residuos de la regresión del modelo de mercado obtenidos por MCO. En consecuencia, se decidió aplicar la prueba de Engle (1982) en los residuos de las 127 regresiones, y se encontraron estos efectos en 16 regresiones del CAR $(-2,2)$, en 18 del CAR $(-3,3)$ y en 23 de CAR $(-5,5)$.

Por esta razón, también se calculan estas regresiones utilizando los modelos $\mathrm{ARCH}$ (1). Se mantienen las regresiones originales por MCO para cada uno de los CAR ya calculados, las cuales no mostraron ningún signo de heterocedasticidad. Las regresiones obtenidas por ARCH han demostrado ser significativamente superiores a los estimados por MCO, en términos de la prueba t, la prueba $\mathrm{F}$ y $\mathrm{R}$ cuadrados.

TABLA 2

Estadisticas descriptivas de las variables del modelo, 2009-2014

\begin{tabular}{|c|c|c|c|c|c|c|c|}
\hline & $N$ & Media & Desv. típ. & Asir & & $\mathrm{Cur}$ & \\
\hline & Estadístico & Estadístico & Estadístico & Estadístico & Error típico & Estadístico & $\begin{array}{l}\text { Error } \\
\text { tipico }\end{array}$ \\
\hline CAR $(-2,2)$ & 127 & 0,0007 & 0,032 & 0,432 & 0,153 & 2,787 & 0,32 \\
\hline CAR $(-3,3)$ & 127 & 0,0001 & 0,024 & 0,218 & 0,153 & 2,151 & 0,32 \\
\hline CAR $(-5,5)$ & 127 & 0,0020 & 0,039 & $-0,783$ & 0,153 & 4,098 & 0,32 \\
\hline D_IRFI & 127 & 0,7782 & 0,289 & $-2,176$ & 0,153 & 2,934 & 0,32 \\
\hline VA & 127 & 0,1782 & 0,247 & 0,236 & 0,153 & 0,298 & 0,32 \\
\hline D_GP & 127 & 0,8387 & 0,135 & $-5,64$ & 0,153 & 39,246 & 0,32 \\
\hline LN (TA) & 127 & 18,8087 & 1,424 & $-1,35$ & 0,153 & 2,932 & 0,32 \\
\hline Div & 127 & 0,1221 & 0,573 & 2,57 & 0,153 & 7,864 & 0,32 \\
\hline Upa & 127 & 0,03091 & 0,184 & 6,079 & 0,153 & 45,084 & 0,32 \\
\hline Apal_f & 127 & 1,0767 & 0,559 & 2,89 & 0,153 & 15,132 & 0,32 \\
\hline Ren_A & 127 & 0,0522 & 0,057 & 1,436 & 0,153 & 3,010 & 0,32 \\
\hline Ln (bm) & 127 & $-0,4801$ & 0,364 & 0,564 & 0,153 & 2,98 & 0,32 \\
\hline deltavol & 127 & $-0,0259$ & 0,139 & $-1,998$ & 0,153 & 19,895 & 0,32 \\
\hline $\begin{array}{l}\text { N válido } \\
\text { (según lista) }\end{array}$ & 127 & & & & & & \\
\hline
\end{tabular}


Así, en el análisis por medio de las estadísticas descriptivas media, desviación estándar, curtosis y simetría (Tabla 1), de cada una de las variables descritas anteriormente, contempla un tamaño de muestra de 127 empresas, durante el periodo 2009-2016. Se puede concluir que las empresas de la muestra en general, sin importar la ventana de evento que se tome, tienen retornos anormales acumulados positivos menores a $1 \%$. También es de observar, que el 87 \% de las empresas tiene informes de revisoría fiscal sin salvedades o limpios, el $97 \%$ de las empresas presenta es sus estados financieros ganancias y solo el $3 \%$ presenta pérdidas.

En consecuencia, el mercado accionario colombiano tiene un buen comportamiento promedio positivo durante el periodo de estudio. Esto podría indicar una relación más fuerte entre los retornos anormales de las empresas y la variable que representa la pérdida y la ganancia, que los mismos informes del revisor fiscal.

\section{Análisis univariante del modelo general}

Analizando las correlaciones que tienen cada una de las variables explicativas con la variable dependiente CAR, la correlación de las variables CAR y D_IRFI (Tabla 2) es positiva en relación con la variable CAR $(-2,2)$, pero es negativa con CAR $(-3,3)$ y CAR $(-5,5)$, pero ninguna de estas correlaciones es significativa ni al $1 \%$ ni al $5 \%$. Esto indicios muestran que los informes del revisor fiscal no tienen impacto en el mercado accionario colombiano. Este análisis también nos indica que los CAR construidos para este estudio se correlacionan de forma significativa con algunas de las variables de control elegidas, lo que muestra que su elección para este estudio es significativa. De hecho, la variable CAR $(-2,2)$ se correlaciona de forma positiva y significativa al $5 \%$ con la variable ganancia perdida, mientras que las otras dos variables dependientes se correlacionan de forma negativa con la variable DeltaVol y significativas ambas al $5 \%$.

Es importante analizar las variables UPA y $\operatorname{Ln}(\mathrm{BM})$, que se correlacionan de forma significativa con otras variables del modelo, más específicamente con la variable D_ IRFI. Estos resultados no permiten asegurar que no hay significancia estadística para que se acepte la hipótesis de investigación, porque los informes de revisoría fiscal no afectan significativamente los retornos de las acciones en el mercado accionario colombiano, durante el periodo de estudio de 2009-2016.

TABLA 3

Correlación de las variables en el modelo general, de 2009 a 2016

\begin{tabular}{|c|c|c|c|c|c|c|c|c|c|c|c|c|c|c|}
\hline & & $\begin{array}{l}\text { CAR } \\
(-2,2)\end{array}$ & $\begin{array}{l}\text { CAR } \\
(-5,5)\end{array}$ & $\begin{array}{l}\text { CAR } \\
(-3,3)\end{array}$ & D_IRFI & VA & D_GP & $\mathrm{LN}(\mathrm{TA})$ & Div & Upa & $\begin{array}{c}\text { Apal } \\
-\mathrm{f}\end{array}$ & Ren_A & $\ln (\mathbf{b m})$ & Deltavol \\
\hline \multirow{3}{*}{ CAR $(-2,2)$} & $\begin{array}{l}\text { Correlación } \\
\text { de Pearson }\end{array}$ & 1 & $0,516^{*}$ & $0,527^{* *}$ & 0,149 & 0,083 & $0,232^{*}$ & 0,029 & 0,024 & $-0,049$ & $-0,197$ & $-0,177$ & 0,007 & $-0,107$ \\
\hline & Sig. (bilateral) & & 0 & 0 & 0,168 & 0,52 & 0,034 & 0,689 & 0,836 & 0,631 & 0,079 & 0,115 & 0,93 & 0,323 \\
\hline & $\mathrm{N}$ & & 127 & 127 & 127 & 127 & 127 & 127 & 127 & 127 & 127 & 127 & 127 & 127 \\
\hline \multirow{3}{*}{ CAR $(-5,5)$} & $\begin{array}{l}\text { Correlación } \\
\text { de Pearson }\end{array}$ & & 1 & $0,747^{* *}$ & $-0,034$ & $-0,033$ & 0,072 & 0,039 & $-0,005$ & 0,082 & $-0,134$ & $-0,185$ & 0,096 & $-0,212^{*}$ \\
\hline & Sig. (bilateral) & & & 0 & 0,615 & 0,61 & 0,392 & 0,781 & 0,943 & 0,398 & 0,204 & 0,059 & 0,372 & 0,035 \\
\hline & $\mathrm{N}$ & & & 127 & 127 & 127 & 127 & 127 & 127 & 127 & 127 & 127 & 127 & 127 \\
\hline \multirow{3}{*}{ CAR $(-3,3)$} & $\begin{array}{l}\text { Correlación } \\
\text { de Pearson }\end{array}$ & & & 1 & $-0,033$ & $-0,001$ & 0,172 & $-0,001$ & $-0,023$ & 0,083 & $-0,041$ & $-0,158$ & 0,056 & $-0,186^{\circ}$ \\
\hline & Sig. (bilateral) & & & & 0,618 & 0.78 & 0,051 & 0,973 & 0,779 & 0,425 & 0.715 & 0,135 & 0.576 & 0.037 \\
\hline & $\mathrm{N}$ & & & & 127 & 127 & 127 & 127 & 127 & 127 & 127 & 127 & 127 & 127 \\
\hline \multirow{4}{*}{ D_IRFI } & $\begin{array}{l}\text { Correlación } \\
\text { de Pearson }\end{array}$ & & & & 1 & 0,19 & 0,165 & 0,039 & 0,181 & $-0,198^{* *}$ & $-0,098$ & 0,084 & $-0,198^{\circ}$ & 0,059 \\
\hline & Sig. (bilateral) & & & & & 0,132 & 0,09 & 0,546 & 0,08 & 0,012 & 0,749 & 0,598 & 0,042 & 0,420 \\
\hline & $\mathrm{N}$ & & & & & 127 & 127 & 127 & 127 & 127 & 127 & 127 & 127 & 127 \\
\hline & $\begin{array}{l}\text { Correlación } \\
\text { de Pearson }\end{array}$ & & & & & 1 & $213^{\circ}$ & 0,0543 & 0,024 & 0,067 & 0,052 & 0,042 & $-0,379^{*}$ & 0,078 \\
\hline
\end{tabular}


TABLA 3 (CONTINUACIÓN)

Correlación de las variables en el modelo general, de 2009 a 2016

\begin{tabular}{|c|c|c|c|c|c|c|c|c|c|c|c|c|c|c|}
\hline & & $\begin{array}{l}\text { CAR } \\
(-2,2)\end{array}$ & $\begin{array}{l}\text { CAR } \\
(-5,5)\end{array}$ & $\begin{array}{l}\text { CAR } \\
(-3,3)\end{array}$ & D_IRFI & va & D_GP & $\operatorname{LN}(\mathrm{TA} A)$ & Dir & Upa & $\begin{array}{l}\text { Apal } \\
\text { ff }\end{array}$ & Ren_A & $\ln (\mathrm{bm})$ & Deltavol \\
\hline \multirow[t]{3}{*}{ va } & Sig. (bilateral) & & & & & & 0,03 & 0,604 & 0,818 & 0,516 & 0,915 & 0,642 & 0 & 0,394 \\
\hline & $\mathrm{N}$ & & & & & & 127 & 127 & 127 & 127 & 127 & 127 & 127 & 127 \\
\hline & $\begin{array}{l}\text { Correlación } \\
\text { de Pearson }\end{array}$ & & & & & & 1 & 0.157 & 0,147 & 0,083 & 0.24 & $0,198^{*}$ & $-0,398^{*}$ & $-0,132$ \\
\hline \multirow{2}{*}{ D_GP } & Sig, (bilateral) & & & & & & & 0,098 & 0,156 & 0,421 & 0,18 & 0,029 & 0 & 0,123 \\
\hline & $\mathrm{s}$ & & & & & & & 127 & 127 & 127 & 127 & 127 & 127 & 127 \\
\hline \multirow[t]{3}{*}{$\operatorname{LN}\left(T_{A} A\right)$} & $\begin{array}{l}\text { Correlación } \\
\text { de Pearson }\end{array}$ & & & & & & & 1 & 0,142 & 0.104 & $-0,102$ & $-0,045$ & $-0.142^{*}$ & 0.178 \\
\hline & Sig. (bilateral) & & & & & & & & 0,169 & 0,316 & 0.532 & 0.849 & 0,023 & 0,312 \\
\hline & $\mathrm{N}$ & & & & & & & & 127 & 127 & 127 & 127 & 127 & 127 \\
\hline \multirow{3}{*}{ Dir } & $\begin{array}{l}\text { Correlación } \\
\text { de Pearson }\end{array}$ & & & & & & & & 1 & 0.057 & -0.098 & 0.063 & $-0,198$ & -0.023 \\
\hline & Sig. (bilateral) & & & & & & & & & 0.584 & 0.309 & 0.515 & 0.397 & 0.924 \\
\hline & $\mathrm{N}$ & & & & & & & & & 127 & 127 & 127 & 127 & 127 \\
\hline \multirow{3}{*}{ Upa } & $\begin{array}{l}\text { Correlación } \\
\text { de Pearson }\end{array}$ & & & & & & & & & 1 & $-0,06$ & $-0,049$ & $-0,042$ & $-0,053$ \\
\hline & Sig. (bilateral) & & & & & & & & & & 0,48 & 0,479 & 0,598 & 0,724 \\
\hline & $\mathrm{N}$ & & & & & & & & & & 127 & 127 & 127 & 127 \\
\hline \multirow{3}{*}{ Apal_ $f$} & $\begin{array}{l}\text { Correlacioin } \\
\text { de Pearson }\end{array}$ & & & & & & & & & & 1 & 0,089 & $-0,053$ & $-0,063$ \\
\hline & Sig. (bilateral) & & & & & & & & & & & 0,43 & 0,378 & 0,62 \\
\hline & $\mathrm{N}$ & & & & & & & & & & & 127 & 127 & 127 \\
\hline \multirow{3}{*}{ Ren_A } & $\begin{array}{l}\text { Correlación } \\
\text { de Pearson }\end{array}$ & & & & & & & & & & & 1 & $-0,359^{*}$ & 0,063 \\
\hline & Sig. (bilateral) & & & & & & & & & & & & 0 & 0,724 \\
\hline & $\mathrm{x}$ & & & & & & & & & & & & 127 & 127 \\
\hline \multirow{3}{*}{ Ln (bm) } & $\begin{array}{l}\text { Correlación } \\
\text { de Pearson }\end{array}$ & & & & & & & & & & & & 1 & 0.009 \\
\hline & Sig. (bilateral) & & & & & & & & & & & & & 0.879 \\
\hline & $\mathrm{N}$ & & & & & & & & & & & & & 127 \\
\hline deltavol & $\begin{array}{c}\text { Correlación } \\
\text { de Pearson } \\
\text { Sig. (bilateral) } \\
\text {. }\end{array}$ & & & & & & & & & & & & & 1 \\
\hline
\end{tabular}

Fuente: elaboración propia. 


\section{Análisis univariante por sectores económicos}

Como en todas las economías, según la literatura, existe una clasificación de los sectores económicos que intervienen en la dinámica del mercado. Para conocer y determinar el impacto que el informe del revisor fiscal ejerce en el mercado accionario se evaluó su efecto en los sectores económicos que intervienen en el mercado accionario colombiano, mediante un análisis descriptivo y un análisis univariado de las variables clasificadas por sectores económicos, que ayudarán a establecer la relación de los informes de revisoría fiscal con el mercado accionario. Se realizó entonces la clasificación en tres sectores, el industrial con 52 empresas, el financiero con 43 y el comercial con 32. Se inició con los análisis descriptivos de cada sector, media y desviación estándar, y luego se pasó a los análisis univariantes (correlaciones) conformados por las variables que mejor se relacionaron con la variable dependiente CAR.

En la Tabla 3 se ilustran los valores promedio y las desviaciones estándar de cada una de las variables del modelo por clasificación sectorial de empresas. Como se puede observar, el sector industrial presenta un retorno normal acumulado promedio negativo en cada una de las tres ventanas de eventos durante el periodo 2009-2016, mientras que los sectores financiero y comercial presentan un retorno normal acumulado promedio positivo.

TABLA 4

Análisis descriptivo por sector

\begin{tabular}{|c|c|c|c|c|c|c|c|c|c|}
\hline Sector & & Industrial & & & Financiero & & & Comercial & \\
\hline Variable & $\mathrm{N}$ & Media & Desv. tip. & $N$ & Media & Desv. tip. & $\mathrm{N}$ & Media & Desv. tip. \\
\hline CAR $(-2,2)$ & 52 & $-0,00476582$ & 0,019583242 & 43 & 0,00423952 & 0,028875629 & 32 & 0,00241404 & 0,022642593 \\
\hline CAR $(-5,5)$ & 52 & $-0,00592123$ & 0,042356310 & 43 & 0,00182874 & 0,016041292 & 32 & 0,01272714 & 0,045200212 \\
\hline $\operatorname{CAR}(-3,3)$ & 52 & $-0,00626512$ & 0,029324398 & 43 & 0,00064529 & 0,029422289 & 32 & 0,00922898 & 0,031250237 \\
\hline D_IRFI & 52 & 0,84 & 0,198 & 43 & 0,83 & 0,209 & 32 & 0,72 & 0,407 \\
\hline VA & 52 & 0,10198356 & 0,236552285 & 43 & 0,12385646 & 0,254082679 & 32 & 0,12343222 & 0,198498098 \\
\hline D_GP & 52 & 0,87 & 0,153 & 43 & 1,00 & 0,000 & 32 & 0,93 & 0,198 \\
\hline LN (TA) & 52 & 21,39843945 & 2,856230914 & 43 & 21,56838329 & 0,920510951 & 32 & 21,43746152 & 1,098514224 \\
\hline Div & 52 & 0,20394256 & 0,821330824 & 43 & 0,09830710 & 0,491324003 & 32 & 0,019972731 & 0,3458734123 \\
\hline Upa & 52 & $-0,00862419$ & 0,235218563 & 43 & 0,01187975 & 0,103243124 & 32 & 0,10524987 & 0,423256098 \\
\hline Apal_f & 52 & 1,08095123 & 0,393221483 & 43 & 1,09735812 & 0,323056012 & 32 & 1,29541714 & 0,789005070 \\
\hline Ren_A & 52 & 0,08279630 & 0,054171836 & 43 & 0,04243785 & 0,028840506 & 32 & 0,03940979 & 0,061004679 \\
\hline Ln (bm) & 52 & $-0,64353742$ & 0,620011432 & 43 & $-0,30043924$ & 0,402006112 & 32 & $-0,40223543$ & 0,429674135 \\
\hline deltavol & 52 & $-0,02837856$ & 0,170665748 & 43 & $-0,01476574$ & 0,029004425 & 32 & $-0,03564082$ & 0,101535785 \\
\hline $\begin{array}{l}\mathrm{N}^{\circ} \text { válido } \\
\text { (según lista) }\end{array}$ & 52 & & & 43 & & & 32 & & \\
\hline
\end{tabular}

Fuente: elaboración propia.

También se puede observar que el sector con menor cantidad promedio $(0,72)$ de informes limpios es el sector comercial, mientras que en el sector industrial el valor promedio es de 0,84 y en el sector financiero es de 0,83 . Así mismo, es importante analizar que el promedio de la variable que representan las ganancias y 
las pérdidas en el sector financiero indica que, en general, el sector financiero del país no presentó pérdidas durante el periodo 2009-2016.

TABLA 5

Correlación en el sector industrial

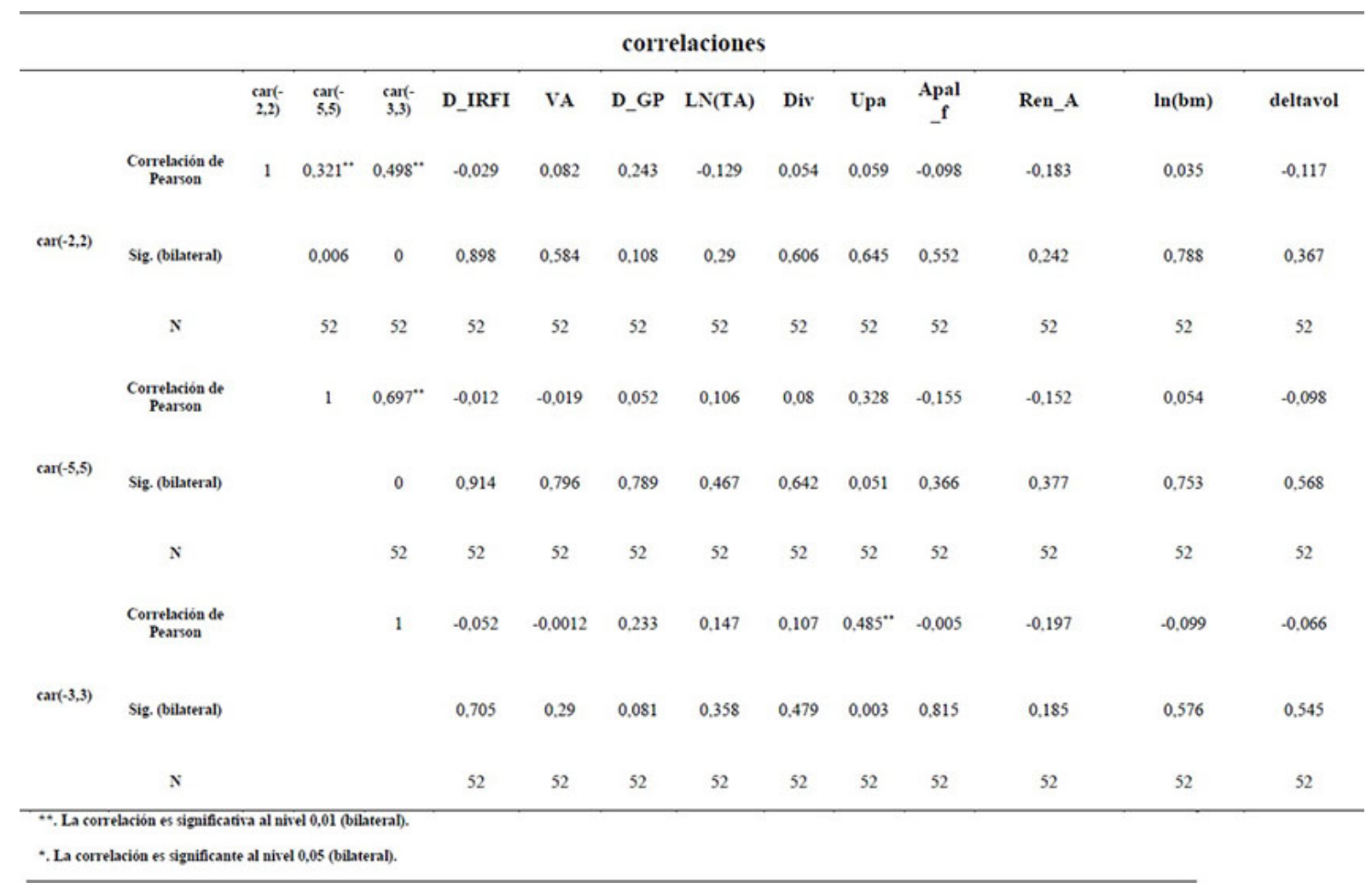

Fuente: elaboración propia.

Ahora se prueba la hipótesis $H_{i}$, analizando las correlaciones que tienen cada una de las variables explicativas con la variable dependiente CAR. Se identificó que al realizar el análisis de correlación por sectores económicos, el sector comercial (Tabla 4) presenta correlaciones existentes entre las variables, como el análisis CAR $(-3,3)$ con las variables Ln (TA) y deltavol a un nivel de significancia del $5 \%$ y el $1 \%$.

TABLA 6

Correlación en el sector financiero

\begin{tabular}{|c|c|c|c|c|c|c|c|c|c|c|c|c|c|c|}
\hline \multicolumn{15}{|c|}{ Correlaciones } \\
\hline & & $\operatorname{car}(-2,2)$ & $\operatorname{car}(-5,5)$ & $\operatorname{car}(-3,3)$ & D_IRFI & VA & D_GP & LN(TA) & Div & Upa & Apal_f & Ren_A & $\ln (\mathrm{bm})$ & deltavol \\
\hline \multirow{3}{*}{$\operatorname{car}(-2,2)$} & $\begin{array}{l}\text { Correlación } \\
\text { de Pearson }\end{array}$ & 1 & $0,602^{*}$ & $0,483^{\prime \prime}$ & 0.149 & 0,064 & & 0.153 & $-0,123$ & -0.135 & $-0,138$ & -0.177 & -0.095 & -0.085 \\
\hline & $\begin{array}{c}\text { Sig. } \\
\text { (bilateral) }\end{array}$ & & 0 & 0.004 & 0.256 & 0.645 & & 0.202 & 0.659 & 0.538 & 0.358 & 0.253 & 0.356 & 0.653 \\
\hline & $\mathrm{N}$ & & 43 & 43 & 43 & 43 & 43 & 43 & 43 & 43 & 43 & 43 & 43 & 43 \\
\hline \multirow{3}{*}{$\operatorname{car}(-5,5)$} & $\begin{array}{l}\text { Correlación } \\
\text { de Pearson }\end{array}$ & & 1 & $00.701^{\prime \prime}$ & 0.143 & 0.086 & & 0.198 & -0.148 & 0.089 & -0.104 & -0.118 & -0.137 & -0.108 \\
\hline & $\begin{array}{c}\text { Sig. } \\
\text { (bilateral) }\end{array}$ & & & 0 & 0.412 & 0.623 & & 0.101 & 0.235 & 0,456 & 0,603 & 0.356 & 0.402 & 0.351 \\
\hline & $\mathrm{N}$ & & & 43 & 43 & 43 & 43 & 43 & 43 & 43 & 43 & 43 & 43 & 43 \\
\hline \multirow{3}{*}{$\operatorname{car}(-3,3)$} & $\begin{array}{l}\text { Correlación } \\
\text { de Pearson }\end{array}$ & & & 1 & 0.096 & 0.029 & & 0.098 & -0.053 & 0.03 & 0.048 & -0.064 & -0.031 & -0.043 \\
\hline & $\begin{array}{c}\text { Sig. } \\
\text { (bilateral) }\end{array}$ & & & & 0.523 & 0.652 & & 0.513 & 0.133 & 0.901 & 0.801 & 0.635 & 0.705 & 0.738 \\
\hline & $\mathrm{N}$ & & & & 43 & 43 & 43 & 43 & 43 & 43 & 43 & 43 & 43 & 43 \\
\hline
\end{tabular}

Fuente: elaboración propia. 
Adicional a esto también encontramos correlación positiva entre las variables deltavol y el CAR $(-5,5)$ a un nivel de significancia del $1 \%$. En este caso se realiza el análisis con un modelo de regresión lineal entre las variables que mejor se correlacionan, teniendo en cuenta que para evitar problemas de multicolinealidad debemos sacar la variable $\operatorname{lm}(\mathrm{BM})$, por su alta correlación significativa con la variable experimental D_IRFI de $-0,53$ a un nivel de significancia del $1 \%$. En este caso solamente se realizó un análisis de las variables por sector industrial, que tiene explicación en los retornos anormales.

TABLA 7

Correlación en el sector comercial

\begin{tabular}{|c|c|c|c|c|c|c|c|c|c|c|c|c|c|c|}
\hline & & \multicolumn{5}{|c|}{ CAR CAR (- CAR (- } & \multicolumn{8}{|c|}{ Apal } \\
\hline & & $(-2,2)$ & $5,5)$ & $3,3)$ & D_IRFI & va & D_GP & $\operatorname{LN}(\mathrm{TA})$ & Div & Upa & $-f$ & Ren_A & Ln (bm) & deltavol \\
\hline \multirow[t]{4}{*}{ CAR $(-2,2)$} & Correlación de & 1 & $0,513^{\prime \prime}$ & $0,551^{\prime \prime}$ & $\overline{0,372}$ & 0,088 & 0,343 & 0,072 & 0,239 & $-0,155$ & $-0,371$ & $-0,074$ & 0,018 & $-0,146$ \\
\hline & Pearson & & & & & & & & & & & & & \\
\hline & Sig. (bilateral) & & 0,002 & 0,008 & 0,088 & 0,697 & 0,118 & 0,752 & 0,285 & 0,490 & 0,089 & 0,742 & 0,936 & 0,516 \\
\hline & $\mathrm{s}$ & & 32 & 32 & 32 & 32 & 32 & 32 & 32 & 32 & 32 & 32 & 32 & 32 \\
\hline \multirow[t]{4}{*}{$\operatorname{CAR}(-5,5)$} & Correlación de & & 1 & $0,921 *$ & $-0,008$ & $-0,271$ & 0,157 & $-0,385$ & 0.067 & $-0,149$ & -0.225 & -0.291 & 0,306 & $-0,609^{\prime \prime}$ \\
\hline & Pearson & & & & & & & & & & & & & \\
\hline & Sig. (bilateral) & & & 0,000 & 0,973 & 0,223 & 0,487 & 0,077 & 0,768 & 0,507 & 0,313 & 0,188 & 0,167 & 0,003 \\
\hline & $\mathrm{s}$ & & & 32 & 32 & 32 & 32 & 22 & 32 & 32 & 32 & 32 & 32 & 32 \\
\hline \multirow[t]{4}{*}{$\operatorname{CAR}(-3,3)$} & Correlación de & & & 1 & 0.000 & $-0,343$ & 0.217 & $-0,457^{\circ}$ & 0,096 & $-0,146$ & $-0,173$ & $-0,172$ & 0,298 & $-0,690^{*}$ \\
\hline & Pearson & & & & & & & & & & & & & \\
\hline & Sig. (bilateral) & & & & 0,998 & 0,118 & 0,331 & 0,033 & 0,670 & 0,516 & 0,440 & 0,445 & 0,179 & 0,000 \\
\hline & $\mathrm{s}$ & & & & 32 & 32 & 32 & 32 & 32 & 32 & 32 & 32 & 32 & 32 \\
\hline \multirow[t]{4}{*}{ D_IRFI } & Correlación de & & & & 1 & 0,345 & 0,319 & $-0,043$ & 0.386 & $-0,314$ & 0.145 & 0,134 & $-0.530^{* *}$ & 0.270 \\
\hline & Pearson & & & & & & & & & & & & & \\
\hline & Sig. (bilateral) & & & & & 0,116 & 0,147 & 0,848 & 0,076 & 0,155 & 0,520 & 0,552 & 0,001 & 0,224 \\
\hline & $\mathrm{s}$ & & & & & 32 & 32 & 32 & 32 & 32 & 32 & 32 & 32 & 32 \\
\hline $\mathrm{vA}$ & Correlación de & & & & & 1 & 0.272 & 0.188 & 0.061 & 0,041 & $-0,020$ & 0.164 & $-0,315$ & 0.268 \\
\hline
\end{tabular}




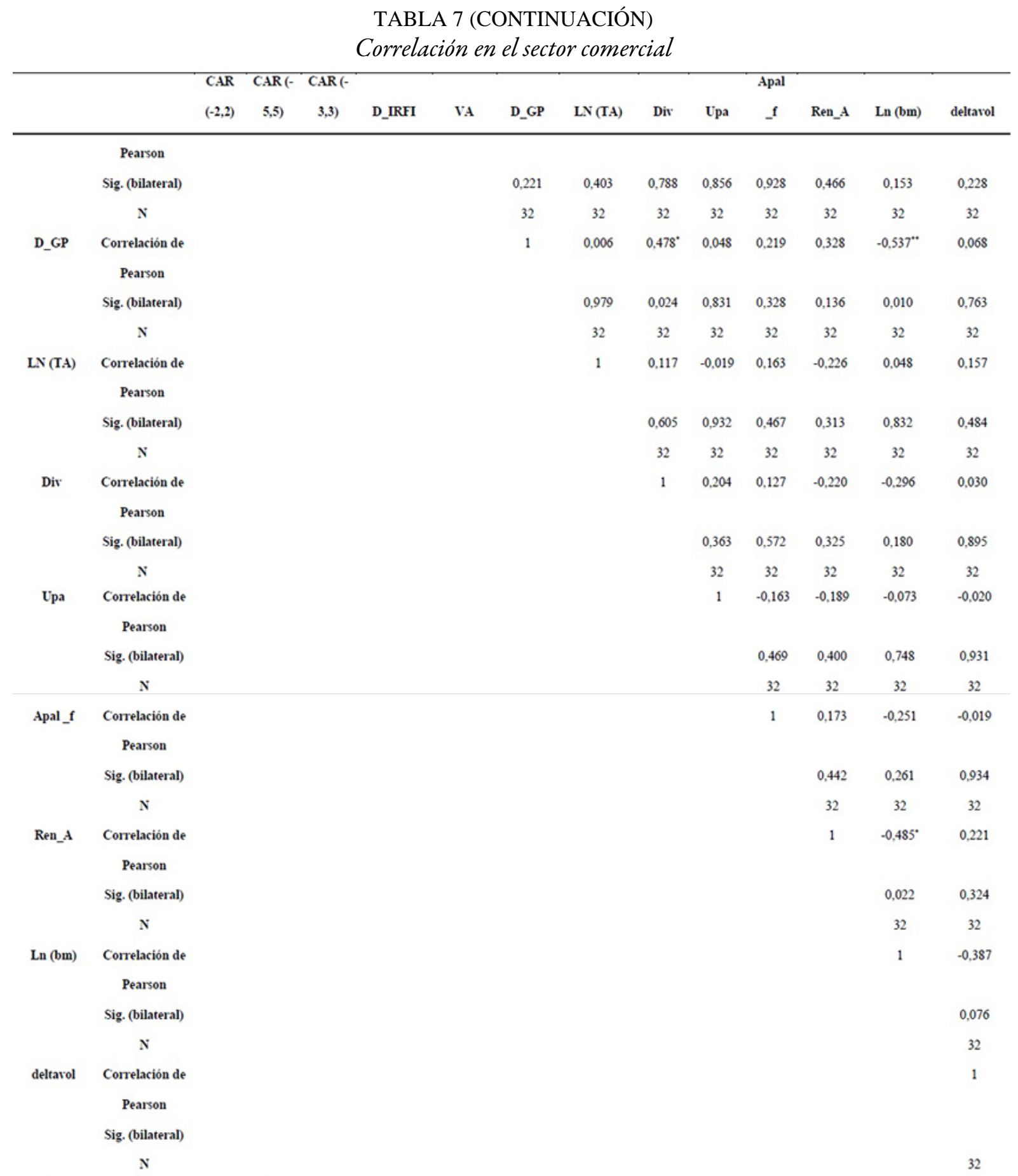

Fuente: elaboración propia. 


\section{Conclusiones}

Esta investigación se realizó dentro de los parámetros de un enfoque cuantitativo con alcance correlacional, utilizando la metodología de estudios de eventos, en la cual se buscó evaluar el efecto que genera un evento en particular, representado por la fecha de divulgación de los informes de revisoría fiscal de las empresas que cotizaron en la Bolsa de Valores de Colombia durante el periodo 2009-2016, buscando medir la volatilidad del precio de las acciones cotizadas durante este lapso, ocasionada por la divulgación del informe del revisor fiscal de las empresas en estudio. Por medio de herramientas estadísticas se dio una aproximación al comportamiento y caracterización de las variables a través de su análisis descriptivo. Estas fueron: 1) la variable dependiente CAR, calculando el retorno anormal acumulado; y 2) la variable D_IRF, considerada como una variable Dummy de los informes de revisoría fiscal; por último, se consideraron ocho variables de control: apalancamiento de las empresas (Apal_f), lucro o pérdidas en las empresas (G_P), tamaño de las empresa [ln (TA)], fluctuación del precio de las acciones (VA), dividendos (Div), utilidad por acción (Upa), rentabilidad (Ren_A) y, por último, volumen de las operaciones (DeltaVol). De esta forma se buscó sustentar o aceptar la hipótesis de investigación propuesta $\left(H_{\mathrm{i}}\right)$, cuyo objetivo era evaluar si la divulgación de los informes de revisoría fiscal de las empresas interfiere en el precio de las acciones que cotizan en la BVC, medida por la volatilidad del precio accionario en una fecha determinada. Igualmente, para darle más robustez a este estudio se realizó un análisis correlacional por medio de un modelo de regresión multivariante y del modelo Egarch, con el fin de evaluar los comportamientos lineales o no lineales de la volatilidad de los precios accionarios.

En consecuencia, se puede concluir que se rechaza la $\mathrm{Hi}$ y se acepta la hipótesis nula $\mathrm{H}_{0}$. Por tanto, el trabajo del revisor fiscal no influye en la dinámica del mercado de valores de Colombia. Para sustentar los resultados anteriores se establecieron unos criterios poblacionales, utilizando un proceso de filtrado de la población mediante el software Economática y la página web de la Superintendencia Financiera de Colombia. Igualmente, con el fin de caracterizar este análisis se buscó por sectores económicos determinar si la divulgación del informe del revisor fiscal provoca un retorno anormal en algún sector económico específico; así se evidenció que en los sectores financiero e industrial no existe ningún tipo de correlación entre la variable CAR y las otras variables analizadas en las tres fechas del evento, principalmente con la variable relacionada del informe del revisor fiscal.

Este estudio brinda evidencia empírica para afirmar que los inversionistas del mercado accionario colombiano no utilizan el informe del revisor fiscal como un factor determinante en la toma de decisiones para participar activamente en este mercado, el cual, con los diferentes análisis estadísticos utilizados, confirmó que el informe del revisor fiscal no interviene en la volatilidad de las acciones del mercado accionario de Colombia.

Podemos indicar que, a pesar de que se utilizaron como variables informaciones de los estados financieros de empresas que en algunos casos fueron analizadas en otros estudios con el mismo objetivo pero en otros contextos económicos, se confirma que la toma de decisiones de los inversionistas no depende de la divulgación de los estados financieros ni del informe del revisor fiscal, lo que deja una puerta abierta para estudios futuros que podrían considerar la incorporación en el modelo de factores macroeconómicos.

Por último, y dando respuesta a uno de los interrogantes que motivó a esta investigación, ¿si diferentes estudios realizados en Europa, Asia y Norteamérica muestran resultados que permiten su generalización o extrapolación a otros mercados, entonces los usuarios del mercado accionario colombiano deben continuar usando esas conclusiones ajenas al contexto colombiano? Podemos indicar que no, principalmente en el caso colombiano, donde contamos con la figura de la revisoría fiscal, que representa una institución de control de los inversionistas y del Estado, que cuenta con una normatividad específica del país y que en muy pocos lugares del mundo existe esta figura de fiscalización y control externo de las entidades. 


\section{Agradecimientos}

Financiado por CODI-Universidad de Antioquia.

\section{Referencias bibliográficas}

Abrantes, R. M. y Dellepiane, S. (2013). La metodología de estudio de eventos en la estimación de daños en casos de arbitraje internacional. Ma de México, Instituto de Investigaciones Jurídicas, (2008), 199-217.

Ameen et al. (1994). Information Content of Qualified Audit Opinions for Over-the-Counter Firms. Journal of Business Finance and Accounting, 21, 997-1011.

Ashley, J. W. (1962). Stock Prices and Changes in Earnings and Dividends: Some Empirical Results. J. Polit. Econ, $70(1), 82-85$.

Ball, R. y Brown, P. (1968). An Empirical Evaluation of Accounting Income Numbers. J. Acc. Res. , Autumn, 6(2), 159-78.

Barker, C. A. (1956). Effective Stock Splits. Harvard Business Review, Vol. 34, No. 1 (January-February, 1956), 101-106.

Barker, C. A. (1957). Stock Splits in a Bull Market. Harvard Business Review, Vol. 35, No. 3 (May-June, 1957), $72-79$.

Barker, C. A. (1958). Evaluation of Stock Dividends. Harvard Business Review, Vol. 36, No. 4 (July-August, 1958), 99-113.

Berggrun, L. (2006). La fusión de Bancolombia, Conavi y Corfinsura: una aplicación de la metodología de estudio de eventos. Estudios Gerenciales, 22 (100), 83-102. Retrieved from: https://www.icesi.edu.co/revistas/index.php/ estudios_gerenciales/article/view/201/html

Bernard, V. L. y Thomas, J. K. (1990). Evidence that Stock Prices do not Fully Reflect the Implications of Current Earnings for Future Earnings. Journal of Accounting and Economics, 13(4), 305-340. https:// doi.org/10.1016/0165-4101(90)90008-R

Black, F. y Scholes, M. (1974). The Effects of Dividend Yields and Dividend Policy on Common Stock Prices and Returns. Journal of Financial Economic, 1, 1-22.

Blanco, M. S. (2003). Auditoría 2005. Bogotá: Ecoe Ediciones.

Bollerslev, T. (1986). Generalized Autoregressive Conditional Heteroscedasticity. Journal of Econometrics, Amsterdam, Holanda.

Bromwich, M. (1992). Financial Reporting, Information and Capital Markets. London: Pitman Publishing.

Chen, C. J. P., Su, X. y Zhao, R. (2000). An Emerging Market's Reaction to Initial Modified Audit Opinions: Evidence from the Shanghai Stock Exchange. Contemporary Accounting Research, 17(3), 429-455. https:// doi.org/10.1506/GCJP-5599-QUWB-G86D

Chiang, T. C. y Doong, S. (2001). Empirical Analysis of Stock Returns and Volatility: Evidence from Seven Asian Stock Markets Based on TAR-GARCH Model. Review of Quantitative Finance and Accounting, Amsterdam, $N E, 17$, nov. 2001, 301-318.

Choi, J. D. (1992). The Effects of Qualified Audit Opinion on Earnings Response Coefficients. Journal of Accounting and Economics, 15 (2), 229-248.

Chow, C. W. y Rice, S. J. (1982). Qualified Audit Opinions and Share Prices: an Investigation. Auditing: A Journal of Practice and Theory, 57, 35-53.

Defond, M. y Wong, T. J. (2000). The Impact of Improved Auditor Independence on Audit Market Concentration in China. Journal of Accounting and Economics, v. 28, 269-305.

Dodd et al. (1984). Qualified Audit Opinions and Stock Prices: Information Content, Announcement Dates, and Concurrent Disclosures. Journal of Accounting and Economics, 6, 3-38.

Dolley, J. C. (1993). Characteristics and Procedure of Common Stock Split-Ups. Harvard Bus. Rev., Apr. 1933, 11, 316-26. 
Elliot, J. (1982). Subject to; Audit Opinions and Abnormal Security Returns Outcomes and Ambiguities. Journal of Accounting Research, 20, 617-638.

Engle, R. F. y Nelson, D. B. (1993). University of California, San Diego, Department of Economics By and Discussion Paper 93-49, (november).

Fama, E. F. (1991). Efficient Capital Markets: Journal of Finance, 46 (5), 383-417. https://doi.org/10.2307/2328565

Fama et al. (1969). The Adjustment Of Stock Prices To New Information. International Economic Review, 10 (1), 1-21. https://doi.org/10.2307/2525569

Firth, M. A. (1978). Qualified Audit Reports: Their Impact on Investment Decisions. Accounting Review, Vol. 53, 642-650.

García, M. A., Ruiz, E. y Vico, A. (2000). Factores que condicionan la elección y el cambio de auditor en la empresa española. Revista de Contabilidad, 3 (6): 49-80.

Gómez, C. (2009). Validación de la hipótesis de los mercados eficientes en su nivel semifuerte aplicada al mercado accionario colombiano. Retrieved from http://www.bdigital.unal.edu.co/2458/1/43983962.2009.pdf

Gutiérrez, B. (2011). A Auditoria e o Mercado Acionário Latino-Americano: Casos Brasil, Argentina e Colômbia. Tesis de doctorado. São Paulo, Universidade de São Paulo, Brasil N. ${ }^{\circ} 177$.

Haw, I. M., Qi, D. y Wu, W. (2000). Timeliness of Annual Report Releases and Market Reaction to Earnings Announcement in an Emerging Capital Market: The Case of China. Journal of International Financial and Accounting, v. 11, 108-131.

Jegadeesh, N. y Livnat, J. (2006). Post-Earnings-Announcement Drift: The Role of Revenue Surprises. Financial Analysts Journal, 62 (2), 22-34.

Jones, F. L. (1996). The Information Content of the Auditor's Going Concern Evaluation. Journal of Accounting and Public Policy, 15, 1-27.

Knechel, R. W. y Sharma, D. S. (2012). Auditor-Provided Nonaudit Services and Audit Effectiveness and Efficiency: Evidence from Pre-and Post-SOX Audit Report Lags. Auditing: A Journal of Practice and Theory, 31 (4), 85-14.

Krishnamurthy et al. (2006). Auditor Reputation, Auditor Independence, and the Stock-Market Impact of Andersen's Indictment on its Client Firms, Contempora, 465-90.

Lin, Z.J., Tang Q. y Xiao J. (2003). An Experimental Study of Users' Responses to Qualified Audit Reports in China. Journal of International Accounting, Auditing and Taxation. v. 12, 1-22.

Loudder et al. (1992). The Information Content of Audit Qualification. Auditing: A Journal of Practice and Theory, $11,69-82$.

MacKinlay, C. (1997). Event Studies in Economics and Finance. Journal of Economic Literature, 35(1), 13-39.

Mantilla, S. A. (2004). Auditoría. Bogotá: Ecoe.

Martínez et al. (2004). Reactions of the Spanish Capital Market to Qualified Audit Reports. European Accounting Review, 13 (4), 689-711. https://doi.org/10.1080/0963818042000216848

Melumad, N. y Ziv, A. (1997). A Theoretical Examination of the Market Reaction to Auditor's Qualifications. Revista de Investigación Contable, 35(2), 239-256.

Myers et al. (1948). Influence of Stock Split-Ups on Market Price. Harvard Bus. Rev, 251-55.

Knechel, R. W. y Sharma, D. S. (2012). Auditor-Provided Nonaudit Services and Audit Effectiveness and Efficiency: Evidence from Evidence from Pre- and Post-SOX. Audit Report Lags., 31, 85-114.

Pei, D., Opong, K., Hamill, P. An Analysis of the Information Content of Qualified Audit Reports: Evidence from China's Shenzhen Market. Working Paper, 2004.

Soltani, B. (2000). Some Empirical Evidence to Support the Relationship Between Audit Reports and Stock PricesThe French Case. International Journal of Auditing, 4(3), 269-291. https://doi.org/10.1111/1099-1123.00317

Teweles et al. (1992). El mercado de valores. 6. Aufl. Nueva York, EE.UU.

Velásquez, G. C. (2009). El impacto de anuncio de eventos en el mercado accionario colombiano. Medellín, Colombia: Universidad Eafit, Escuela de Administración y Finanzas. 


\section{Notas}

[1] Concepto No. 1998063805-1, del 22 de enero de 1999. Secretario General. Declaración profesional N. ${ }^{\circ}$ del Consejo Técnico de la Contaduría Pública.

\section{Licencia Creative Commons CC BY 4.0}

Para citar este artículo: Gutiérrez-Castañeda, B. E. y Barrera-Montoya, C. A. (2018). Metodología de estudio de eventos como medición del impacto del dictamen del revisor fiscal en el mercado accionario colombiano en 2009-2016. Cuadernos de Contabilidad, 19(47), 149-170. https://doi.org/10.11144/ Javeriana.cc19-47.meem 